Research Paper

International Journal of Medical Sciences

ISSN 1449-1907 www.medsci.org 2008 5(2):50-61

(C) Ivyspring International Publisher. All rights reserved

\title{
Computerized two-lead resting ECG analysis for the detection of coronary artery stenosis aiter coronary revascularization
}

\author{
Eberhard Grube1, Andreas Bootsveld², Lutz Buellesfeld1', Seyrani Yuecel ${ }^{1}$, Joseph T Shen³, Michael Imhoff ${ }^{4}$
}

1. Department of Cardiology and Angiology, HELIOS Heart Center Siegburg, Siegburg, Germany

2. Department of Cardiology, Evangelisches Stift St. Martin, Koblenz, Germany

3. Premier Heart, LLC, Port Washington, NY, USA

4. Department for Medical Informatics, Biometrics and Epidemiology, Ruhr-University Bochum, Bochum, Germany

Correspondence to: Michael Imhoff, MD, PhD, Am Pastorenwäldchen 2, D-44229 Dortmund, Germany. Phone: +49-231-973022-0; Fax: +49-231-973022-31; e-mail: mike@imhoff.de

Received: 2007.12.10; Accepted: 2008.03.02; Published: 2008.03.02

Background: Resting electrocardiogram (ECG) shows limited sensitivity and specificity for the detection of coronary artery disease (CAD), where patients with a history of coronary revascularization may pose special challenges. Several methods exist to enhance sensitivity and specificity of resting ECG for diagnosis of CAD, but such methods are not better than a specialist's judgement. We compared a new computer-enhanced, resting ECG analysis device, $3 \mathrm{DMP}$, to coronary angiography to evaluate the device's accuracy in detecting hemodynamically relevant CAD.

Methods: A convenience sample of 172 patients with a history of coronary revascularization scheduled for coronary angiography was evaluated with 3DMP before coronary angiography. 3DMP's sensitivity and specificity in detecting hemodynamically relevant coronary stenosis as diagnosed with coronary angiography were calculated as well as odds ratios for the 3DMP severity score and coronary artery disease risk factors.

Results: The 3DMP system accurately identified 50 of 55 patients as having hemodynamically relevant stenosis (sensitivity $90.9 \%$, specificity $88.0 \%$ ). Positive and negative predictive values for the identification of coronary stenosis as diagnosed in coronary angiograms were $62.7 \%$ and $97.8 \%$ respectively. Risk and demographic factors in a logistic regression model had a markedly lower predictive power for the presence of coronary stenosis in these patients than did 3DMP severity score (odds ratio 2.04 [0.74-5.62] vs. 73.57 [25.10-215.68]). A logistic regression combining severity score with risk and demographic factors did not add significantly to the prediction quality (odds ratio 80.00 [27.03-236.79]).

Conclusions: 3DMP's computer-based, mathematically derived analysis of resting two-lead ECG data provides detection of hemodynamically relevant CAD in patients with a history of coronary revascularization with high sensitivity and specificity that appears to be at least as good as those reported for other resting and/or stress ECG methods currently used in clinical practice.

Key words: coronary artery disease, electrocardiography, computer-enhanced, coronary imaging: angiography, sensitivity, specificity, post-intervention

\section{Introduction}

Coronary artery disease (CAD) is the leading single cause of death in the developed world. Between $15 \%$ and $20 \%$ of all hospitalizations are the direct results of CAD [1].

Revascularization of coronary arteries is one of the most frequently performed medical interventions in the developed world. In 2002, more than 500,000 coronary artery bypass graft (CABG) surgeries and nearly 1.2 million percutaneous coronary interventions (PCI) including coronary stent implantations were performed in the US. In the same year, more than 200,000 CABGs and more than half a million PCI were done in Europe [1]. Coronary restenosis after PCI and bypass graft and de-novo coronary stenosis are not infrequent after revascularization and remain significant clinical issues [2]. For example, studies of drug eluting and non-drug eluting stents show restenosis rates between $4 \%$ and over $20 \%[3,4]$.

Coronary angiography remains the gold standard for the morphologic diagnosis of CAD and also allows revascularization during the same procedure $[5,6]$. However, it is resource-intensive, expensive, invasive, and bears a relevant procedure-related complication rate $(<2 \%)$, morbidity $(0.03-0.25 \%)$, and mortality $(0.01-0.05 \%)[7,8]$.

Electrocardiography-based methods are routinely 
used as the first tools for initial screening and diagnosis. Still, in clinical studies they show sensitivities for prediction of CAD of only $20 \%$ to $70 \%$ $[9,10]$. Even exercise ECG is an insensitive method to detect restenosis, with a sensitivity of below $55 \%$. Therefore, the usefulness of ECG-based methods in the follow-up period after revascularization therapy has been questioned [11, 12, 13].

Risk factors for CAD such as smoking, arterial hypertension, diabetes mellitus, obesity, or hypercholesterolemia (of which at least one is present in the vast majority of symptomatic CAD patients) can also be used to screen for hemodynamically relevant coronary stenosis $[14,15,16,17]$. But in patients after coronary revascularization these risk factors are often modified by secondary prevention and have not been well validated for establishing pre-test probability of coronary stenosis.

Several methods have been proposed and developed to enhance sensitivity and specificity of resting ECG for diagnosis of symptomatic and asymptomatic CAD. Yet such diagnostic ECG computer programs have not been shown to be equal or superior to the specialist physician's judgment [18]. Moreover, studies comparing computerized with manual ECG measurements in patients with acute coronary syndrome have shown that computerized measurements have diagnostic cut-offs that differ from manual measurements and may not be used interchangeably [19]. This is likely one reason underlying the limited acceptance of such techniques in clinical practice during the follow-up period after coronary revascularization.

The present study compared 3DMP, a new computer-enhanced, resting ECG device, to coronary angiography to evaluate $3 \mathrm{DMP}^{\prime} \mathrm{s}$ relevance in detecting coronary restenosis, graft stenosis, or de novo stenosis after coronary revascularization.

\section{Methods and Materials}

The study was approved by the local institutional committee on human research. Written informed consent was waived by each participant as a result of the disclosed non-risk designation of the study device. All patients received a full explanation and gave verbal consent to the study and the use of their de-identified data.

\section{Patients}

Between July 01, 2001, and June 30, 2003, 213 patients scheduled for coronary angiography at the Heart Center Siegburg, Siegburg, Germany, were included in the study. These patients represented a convenience sample in that each patient was already scheduled for coronary angiography for any indication and had undergone at least one coronary revascularization procedure at least 6 weeks prior to the scheduled angiography. Thirty-six patients had a history of myocardial infarction (MI) more than six weeks prior to angiography. No patients presented with acute coronary syndrome at the time of study. Seven patients were excluded from the final analysis due to poor ECG tracing quality, and risk factor information could not be retrieved for 34 patients. The patient population did not overlap with any previous study or with the actual 3DMP database. The 3DMP reference database was not modified or updated during the study period.

Medical history and risk factors for each patient were retrieved from the standard medical documentation. The following risk factors were classified as "present" or "not present" [14, 15, 16, 17]:

- Arterial hypertension (systolic blood pressure > $140 \mathrm{mmHg}$ and/or diastolic blood pressure $>90$ $\mathrm{mmHg}$ ),

- Diabetes mellitus of any type,

- Hypercholesterolemia (cholesterol $>200 \mathrm{mg} / \mathrm{dl}$ or LDL-cholesterol > $160 \mathrm{mg} / \mathrm{dl}$ ) and/or hypertriglyceridemia (triglycerides $>200 \mathrm{mg} / \mathrm{dl}$ ),

- Active or former smoking (cessation less than 5 years prior to inclusion in the study),

- Obesity (BMI $>30 \mathrm{~kg} / \mathrm{m} 2)$,

- Family history (symptomatic CAD of one parent),

- Other risk factors, including established diagnosis of peripheral artery disease.

\section{Study device}

The study device, 3DMP (Premier Heart, LLC, Port Washington, NY, USA), records a 2-lead resting ECG from leads II and V5 for 82 seconds each using proprietary hardware and software. The analog ECG signal is amplified, digitized, and down-sampled to a sampling rate of $100 \mathrm{~Hz}$ to reduce data transmission size; subsequent data transformations performed on the data do not require higher than $100 \mathrm{~Hz} / \mathrm{sec}$ resolution. The digitized ECG data is encrypted and securely transmitted over the Internet to a central server.

At the server, a series of Discrete Fourier Transformations are performed on the data from the two ECG leads followed by signal averaging. The final averaged digital data segment is then subjected to six mathematical transformations (power spectrum, coherence, phase angle shift, impulse response, cross-correlation, and transfer function) in addition to an amplitude histogram, all of which is used to 
generate indexes of abnormality. The resulting patterns of the indexes are then compared for abnormality to the patterns in the reference database to reach a final diagnostic output. In addition to the automatic differential diagnosis and based on the database comparison, a severity score from 0 to 20 is calculated that indicates the level of myocardial ischemia (if present) resulting from coronary disease.

The database against which the incoming ECG results are compared originated from data gathering trials conducted from 1978 to 2000 in more than 30 institutions in Europe, Asia, and North America on individuals of varying ages and degrees of disease state including normal populations [20,21]. All ECG analyses in this database have been validated against the final medical diagnosis of at least two independent expert diagnosticians in the field, including results of angiography and enzyme tests. The current diagnostic capability for identification of local or global ischemia and the disease severity score used in this clinical study are based on 3DMP's large proprietary database of validated ECG analyses accumulated since 1998.

One important difference between 3DMP and other ECG methods is that the ECG is locally recorded but remotely analyzed at a central data facility due to the size and complexity of the reference database. A detailed description of the 3DMP technology was given previously in this journal [22].

\section{ECG acquisition and processing}

3DMP tests were conducted as follows by a trained trial site technician as part of a routine electrophysiological workup received by each patient prior to angiography.

Patients were tested while quietly lying supine following 20 minutes of bed rest.

Five ECG wires with electrodes were attached from the 3DMP machine to the patient at the four standard limb lead and precordial lead V5 positions.

An automatic 82-second simultaneous two-lead (leads V5 and II) ECG sample was acquired with amplification and digitization.

During the sampling, the ECG tracings displayed on the 3DMP screen were closely monitored for tracing quality.

The digital data was then de-identified, encrypted, and sent via a secure Internet connection to www.premierheart.com. A second identical copy of the data was saved on the remote 3DMP machine for post-study verification purposes before the data analysis was carried out. The quality of the tracing was visually rechecked and graded as "good," "marginal," or "poor." A poor tracing was defined by one of the following:

- five or more 5.12-second segments of ECG data contain idiopathic extrema that deviate from the baseline by $\geq 2 \mathrm{~mm}$ and appear $\geq 10$ times,

- two or more 5.12-second segments of ECG data contain idiopathic extrema that deviate from the baseline by $\geq 5 \mathrm{~mm}$,

- in a 25-mm section of waveform in any 5.12-second segment of the ECG data, the waveform strays from the baseline by $\geq 3 \mathrm{~mm}$,

- a radical deviation away from the baseline $80^{\circ}$ of $\geq$ $2 \mathrm{~mm}$ from the baseline, occurring two or more times,

- a single radical deviation away from the baseline $80^{\circ}$ episode of $\geq 5 \mathrm{~mm}$ from the baseline.

A marginal tracing was defined by significant baseline fluctuations that did not meet the above criteria. Tracings consistently graded as poor after repeated sampling were excluded from the present study. All other tracings were included in the study.

3DMP provided automatic diagnosis of regional or global ischemia, including silent ischemia, due to coronary artery disease, and calculated a severity score. This severity score has a maximum range from 0 to 20 where a higher score indicates a higher likelihood of myocardial ischemia due to coronary stenosis. Following the 3DMP manufacturer's recommendation, a cut-off of 4.0 for the severity score was used in this study, with a score of 4.0 or higher being considered indicative of a hemodynamically relevant coronary artery stenosis of $>70 \%$ in at least one large-sized vessel.

Angiographers and staff at the study site were blinded to all 3DMP findings. The 3DMP technicians and all Premier Heart staff were blinded to all clinical data including pre-test probabilities for CAD or angiography findings from the study patients.

Retest reliability of 3DMP was assessed in 38 patients on whom a second 3DMP test was done within 4 hours after the first test. The ECG electrodes were left in place for these repeat measurements. For comparison with angiography, the first test was always used in these patients.

\section{Angiography}

After the 3DMP test, coronary angiography was performed following the standards of the institution. Angiograms were classified immediately by the respective angiographer and independently by a second interventional cardiologist within 4 weeks after the angiogram. If the two investigators did not agree on the results, they discussed the angiograms until agreement was reached. Angiograms were classified as 
follows:

Non-obstructive CAD: angiographic evidence of coronary arterial stenosis of $\leq 70 \%$ in a single or multiple vessels. Evidence included demonstrable vasospasm, delayed clearance of contrast medium indicating potential macro- or micro-vascular disease, documented endothelial abnormality (as indicated by abnormal contrast staining), or CAD with at least $40 \%$ luminal encroachment observable on angiograms. These patients were classified as negative for hemodynamically relevant CAD (= "stenosis: no").

Obstructive CAD: angiographic evidence of coronary arterial sclerosis of $>70 \%$ in a single or multiple vessels, with the exception of the left main coronary artery, where $\geq 50 \%$ was considered obstructive. These patients were classified as positive for hemodynamically relevant CAD (= "stenosis: yes").

The angiographic results represent the diagnostic endpoint against which 3DMP was tested.

\section{Statistical methods}

An independent study monitor verified the double-blindness of the study and the data integrity and monitored the data acquisition process, all angiography reports, and all 3DMP test results. Descriptive statistics were calculated for all variables (mean +/- standard deviation). Differences between two variables were tested with the $t$-test. Differences in $2 \times 2$ tables were assessed for significance with Fisher's exact test. Logistic regression was used to analyze effects of multiple categorical variables. Odds ratios including 95\% confidence intervals were calculated. Sensitivity and specificity were calculated as were receiver operating characteristic (ROC) curves including an estimate of the area under the curve (AUC). Positive and negative predictive values (PPV, NPV) for the assessment of coronary stenosis were calculated with adjustment to prevalence of stenosis [23]. Moreover, in order to assess the performance of the prediction of stenosis independent of the prevalence of stenosis the positive and negative likelihood ratios (LR) were calculated [24]. A value of $P$ $<0.05$ was considered statistically significant. All analyses were done with SPSS for Windows Version 14 (SPSS Inc., Chicago, IL, USA).

\section{Results}

Data from 172 of the original 213 patients were available for final analysis. The 41 patients excluded due to poor ECG tracings (7) or unavailability of full risk factor information (34) were not significantly different from the included patients with respect to age
$(63.7+/-9.1$ years vs. $63.9+/-10.0$ years; $p=0.925)$, gender $(29.3 \%$ female vs.32.6\% male; $p=0.852)$, diagnosis of coronary stenosis ( $39 \%$ vs. $32 \%$; $p=0.461)$, and type of revascularization procedure (CABG $41.5 \%$ CABG vs. $28.5 \% ; p=0.132)$. The study patients comprised 116 men and 56 women, with an average age of $63.9+/-10$ years (35-83). Women were significantly older than men $(68.7+/-8.2$ years vs. 61.6 +/- 9.9 years; $\mathrm{p}<0.01$ ).

Forty-nine patients underwent CABG surgery and $123 \mathrm{PCI}$ prior to angiography. Men undergoing PCI were significantly younger than men undergoing CABG $(60.0+/-10$ years vs. $64.7+/-9.2$ years, $p<0.02$; table 1$)$. In the PCI patients, women were significantly older than men $(69.3+/-7.6$ years vs. $60.0+/-10$ years, $\mathrm{p}<0.01)$, whereas there was no significant age difference in the CABG patients $(66.0+/-10.6$ years vs. $64.7+/-9.2$ years, $\mathrm{p}=0.725)$.

Only $7(4.1 \%)$ patients had no known risk factors for CAD, whereas 103 (59.9\%) had at least three risk factors (table 1). Patients with arterial hypertension and with a family history of CAD were significantly older than those without; smokers were significantly younger than non-smokers (each $\mathrm{p}<0.05$ ). Diabetes was significantly more frequent in women $(p<0.05)$.

Hemodynamically relevant coronary or graft stenosis was diagnosed by angiography in 55 patients $(32 \%)$. There were no significant differences between men and women in the rate of stenosis. There were also no significant age differences between patients with and patients without stenosis (table 2). The percentage of angiographically identified stenosis was higher in the CABG group than in the PCI group, but not significantly $(40.8 \%$ vs. $28.5 \%$; $p=0.15)$. Of the 36 patients with a history of myocardial infarction only 15 $(42 \%)$ had a hemodynamically relevant stenosis. The difference to patients without an MI history was not statistically significant.

In a logistic regression model with all risk factors, age, gender, the type of revascularization procedure, only arterial hypertension was negatively associated with an increase in the risk of coronary stenosis (OR 0.34 [0.16-0.72]; $\mathrm{p}<0.01$ ). A weak, but not significant, association could be seen with CABG (OR 1.86 [0.88-3.93]; $p=0.10$ ). With this model, $67.4 \%$ of all cases were correctly classified (OR 2.04 [0.74-5.62], summary in table 3). When history of MI was included in this model, the model did not significantly change. Specifically, history of MI was not a significant factor in this model. 
Table 1: Risk factors, gender, age distribution, type of revascularization, and MI history.

\begin{tabular}{|c|c|c|c|c|c|c|c|c|c|c|c|c|c|}
\hline & \multicolumn{4}{|c|}{ All Patients } & \multicolumn{8}{|c|}{ Gender } \\
\hline & & & & \multirow[b]{3}{*}{$\mathbf{N}$} & \multirow[b]{3}{*}{$\%$} & \multicolumn{4}{|c|}{ female } & \multicolumn{4}{|c|}{ male } \\
\hline & & \multicolumn{2}{|c|}{ Age (years) } & & & \multicolumn{2}{|c|}{ Age (years) } & \multirow[b]{2}{*}{$\mathbf{N}$} & \multirow[b]{2}{*}{$\%$} & \multicolumn{2}{|c|}{ Age (years) } & \multirow[b]{2}{*}{$\mathbf{N}$} & \multirow[b]{2}{*}{$\%$} \\
\hline & & Mean & SD & & & Mean & SD & & & Mean & SD & & \\
\hline \multirow{2}{*}{$\begin{array}{c}\text { Arterial } \\
\text { Hypertension }\end{array}$} & no & 61.0 & 10.3 & 44 & $25.6 \%$ & 63.9 & 8.3 & 11 & $19.6 \%$ & 60.1 & 10.9 & 33 & $28.4 \%$ \\
\hline & yes & 64.9 & 9.7 & 128 & $74.4 \%$ & 69.8 & 7.9 & 45 & $80.4 \%$ & 62.2 & 9.6 & 83 & $71.6 \%$ \\
\hline \multirow{2}{*}{$\begin{array}{l}\text { High } \\
\text { Cholesterol/Lipids }\end{array}$} & no & 64.9 & 9.1 & 51 & $29.7 \%$ & 70.2 & 9.5 & 14 & $25.0 \%$ & 62.9 & 8.2 & 37 & $31.9 \%$ \\
\hline & yes & 63.4 & 10.3 & 121 & $70.3 \%$ & 68.1 & 7.8 & 42 & $75.0 \%$ & 60.9 & 10.6 & 79 & $68.1 \%$ \\
\hline \multirow{2}{*}{$\begin{array}{l}\text { Active or Former } \\
\text { Smoking }\end{array}$} & no & 66.2 & 9.9 & 105 & $61.0 \%$ & 70.4 & 8.0 & 39 & $69.6 \%$ & 63.7 & 10.1 & 66 & $56.9 \%$ \\
\hline & yes & 60.3 & 9.0 & 67 & $39.0 \%$ & 64.6 & 7.4 & 17 & $30.4 \%$ & 58.8 & 9.1 & 50 & $43.1 \%$ \\
\hline \multirow{2}{*}{$\begin{array}{l}\text { Diabetes of any } \\
\text { type }\end{array}$} & no & 63.5 & 10.2 & 131 & $76.2 \%$ & 68.9 & 8.7 & 37 & $66.1 \%$ & 61.3 & 10.0 & 94 & $81.0 \%$ \\
\hline & yes & 65.3 & 9.3 & 41 & $23.8 \%$ & 68.2 & 7.5 & 19 & $33.9 \%$ & 62.7 & 10.0 & 22 & $19.0 \%$ \\
\hline \multirow[t]{2}{*}{ Family History } & no & 66.1 & 9.6 & 109 & $63.4 \%$ & 71.5 & 8.0 & 32 & $57.1 \%$ & 63.9 & 9.4 & 77 & $66.4 \%$ \\
\hline & yes & 60.0 & 9.4 & 63 & $36.6 \%$ & 64.8 & 7.0 & 24 & $42.9 \%$ & 57.0 & 9.6 & 39 & $33.6 \%$ \\
\hline \multirow[t]{2}{*}{ Obesity } & no & 64.5 & 9.5 & 100 & $58.1 \%$ & 68.2 & 8.7 & 30 & $53.6 \%$ & 63.0 & 9.5 & 70 & $60.3 \%$ \\
\hline & yes & 63.0 & 10.6 & 72 & $41.9 \%$ & 69.2 & 7.8 & 26 & $46.4 \%$ & 59.5 & 10.4 & 46 & $39.7 \%$ \\
\hline \multirow[t]{2}{*}{ Other Risk Factors } & no & 63.8 & 10.0 & 168 & $97.7 \%$ & 68.7 & 8.2 & 56 & $100.0 \%$ & 61.4 & 10.0 & 112 & $96.6 \%$ \\
\hline & yes & 66.5 & 7.0 & 4 & $2.3 \%$ & & & & & 66.5 & 7.0 & 4 & $3.4 \%$ \\
\hline \multirow{7}{*}{$\begin{array}{c}\text { Number of Risk } \\
\text { Factors }\end{array}$} & 0 & 67.1 & 8.6 & 7 & $4.1 \%$ & & & & & 67.1 & 8.6 & 7 & $6.0 \%$ \\
\hline & 1 & 66.7 & 9.3 & 20 & $11.6 \%$ & 73.3 & 6.1 & 6 & $10.7 \%$ & 63.8 & 9.1 & 14 & $12.1 \%$ \\
\hline & 2 & 64.7 & 10.3 & 42 & $24.4 \%$ & 68.6 & 8.9 & 15 & $26.8 \%$ & 62.6 & 10.6 & 27 & $23.3 \%$ \\
\hline & 3 & 62.8 & 10.4 & 54 & $31.4 \%$ & 68.3 & 10.5 & 15 & $26.8 \%$ & 60.7 & 9.7 & 39 & $33.6 \%$ \\
\hline & 4 & 66.8 & 8.9 & 22 & $12.8 \%$ & 70.3 & 7.4 & 10 & $17.9 \%$ & 63 & 9.3 & 12 & $10.3 \%$ \\
\hline & 5 & 59.1 & 8.7 & 20 & $11.6 \%$ & 65.4 & 3.5 & 8 & $14.3 \%$ & 54.8 & 8.7 & 12 & $10.3 \%$ \\
\hline & 6 & 60.6 & 10.2 & 7 & $4.1 \%$ & 63.0 & 1.4 & 2 & $3.6 \%$ & 59.6 & 12.3 & 5 & $4.3 \%$ \\
\hline \multirow{2}{*}{$\begin{array}{l}\text { Myocardial } \\
\text { infarction in } \\
\text { history }\end{array}$} & no & 64.1 & 9.4 & 136 & $79.1 \%$ & 68.2 & 8.2 & 46 & $82.1 \%$ & 62.1 & 9.3 & 90 & $77.6 \%$ \\
\hline & yes & 62.9 & 12.0 & 36 & $20.9 \%$ & 70.8 & 8.7 & 10 & $17.9 \%$ & 59.9 & 11.9 & 26 & $22.4 \%$ \\
\hline \multirow{2}{*}{$\begin{array}{l}\text { Revascularization } \\
\text { in Patient History }\end{array}$} & PCI & 63.4 & 10.2 & 123 & $71.5 \%$ & 69.3 & 7.6 & 45 & $80.4 \%$ & 60.0 & 10.0 & 78 & $67.2 \%$ \\
\hline & CABG & 65.0 & 9.4 & 49 & $28.5 \%$ & 66.0 & 10.6 & 11 & $19.6 \%$ & 64.7 & 9.2 & 38 & $32.8 \%$ \\
\hline
\end{tabular}

Table 2: Frequency of coronary stenosis, distribution of gender, age, type of revascularization, risk factors, and MI history.

\begin{tabular}{|c|c|c|c|c|c|c|}
\hline & & & & \multicolumn{2}{|c|}{ Coronary Stenosis } & \multirow{2}{*}{ All Patients } \\
\hline & & & & no & yes & \\
\hline \multirow[t]{3}{*}{ All Patients } & \multicolumn{3}{|c|}{ Mean } & 63.9 & 63.9 & 63.9 \\
\hline & \multicolumn{3}{|c|}{ Std Deviation } & 9.5 & 11.0 & 10.0 \\
\hline & \multicolumn{3}{|c|}{$\mathrm{N}$} & 117 & 55 & 172 \\
\hline \multirow[t]{6}{*}{ Gender } & female & Age (years) & Mean & 69.3 & 67.3 & 68.7 \\
\hline & & & SD & 7.7 & 9.5 & 8.2 \\
\hline & & & $\mathrm{N}$ & 39 & 17 & 56 \\
\hline & male & Age (years) & Mean & 61.2 & 62.4 & 61.6 \\
\hline & & & SD & 9.2 & 11.4 & 9.9 \\
\hline & & & $\mathrm{N}$ & 78 & 38 & 116 \\
\hline \multirow[t]{2}{*}{ Arterial Hypertension } & no & \multicolumn{2}{|l|}{$\mathrm{N}$} & 22 & 22 & 44 \\
\hline & yes & \multicolumn{2}{|l|}{$\mathrm{N}$} & 95 & 33 & 128 \\
\hline \multirow{2}{*}{ High Cholesterol/Lipids } & no & \multicolumn{2}{|l|}{$\mathrm{N}$} & 32 & 19 & 51 \\
\hline & yes & \multicolumn{2}{|l|}{$\mathrm{N}$} & 85 & 36 & 121 \\
\hline \multirow[t]{2}{*}{ Active or Former Smoking } & no & \multicolumn{2}{|l|}{$\mathrm{N}$} & 72 & 33 & 105 \\
\hline & yes & \multicolumn{2}{|l|}{$\mathrm{N}$} & 45 & 22 & 67 \\
\hline \multirow{2}{*}{ Diabetes of any type } & no & \multicolumn{2}{|l|}{$\mathrm{N}$} & 89 & 42 & 131 \\
\hline & yes & \multicolumn{2}{|l|}{$\mathrm{N}$} & 28 & 13 & 41 \\
\hline \multirow[t]{2}{*}{ Family History } & no & \multicolumn{2}{|l|}{$\mathrm{N}$} & 73 & 36 & 109 \\
\hline & yes & \multicolumn{2}{|l|}{$\mathrm{N}$} & 44 & 19 & 63 \\
\hline \multirow[t]{2}{*}{ Obesity } & no & \multicolumn{2}{|l|}{$\mathrm{N}$} & 68 & 32 & 100 \\
\hline & yes & \multicolumn{2}{|l|}{$\mathrm{N}$} & 49 & 23 & 72 \\
\hline \multirow[t]{2}{*}{ Other Risk Factors } & no & \multicolumn{2}{|l|}{$\mathrm{N}$} & 114 & 54 & 168 \\
\hline & yes & \multicolumn{2}{|l|}{$\mathrm{N}$} & 3 & 1 & 4 \\
\hline \multirow[t]{5}{*}{ Number of Risk Factors } & 0 & \multicolumn{2}{|l|}{$\mathrm{N}$} & 4 & 3 & 7 \\
\hline & 1 & \multicolumn{2}{|l|}{$\mathrm{N}$} & 10 & 10 & 20 \\
\hline & 2 & $\mathrm{~N}$ & & 32 & 10 & 42 \\
\hline & 3 & $\mathrm{~N}$ & & 37 & 17 & 54 \\
\hline & 4 & $\mathrm{~N}$ & & 12 & 10 & 22 \\
\hline
\end{tabular}




\begin{tabular}{|c|c|c|c|c|c|}
\hline & & & \multicolumn{2}{|c|}{ Coronary Stenosis } & \multirow[t]{2}{*}{ All Patients } \\
\hline & & & no & yes & \\
\hline & 5 & $\mathrm{~N}$ & 16 & 4 & 20 \\
\hline & 6 & $\mathrm{~N}$ & 6 & 1 & 7 \\
\hline \multirow{2}{*}{ Myocardial infarction in history } & no & $\mathrm{N}$ & 96 & 40 & 136 \\
\hline & yes & $\mathrm{N}$ & 21 & 15 & 36 \\
\hline \multirow{2}{*}{ Revascularization in Patient History } & PCI & $\mathrm{N}$ & 88 & 35 & 123 \\
\hline & CABG & $\mathrm{N}$ & 29 & 20 & 49 \\
\hline
\end{tabular}

Table 3: Prediction of coronary stenosis by logistic regression with risk factors ("A"), by logistic regression with risk factors and MI history ("B"), by logistic regression with risk factors and severity score (cut-off 4.0; "C"), by logistic regression with risk factors and MI history and severity score (cut-off 4.0; "D"), and by severity score (cut-off 4.0; "E") alone for total population, gender, age groups, type of revascularization, and MI history.

\begin{tabular}{|c|c|c|c|c|c|c|c|c|c|c|c|c|c|c|c|c|c|c|c|c|}
\hline & & \multirow[t]{2}{*}{$\mathbf{n}$} & \multirow[t]{2}{*}{ TP } & \multirow[t]{2}{*}{ TN I } & \multirow{2}{*}{\multicolumn{3}{|c|}{ FP FN a priori }} & \multirow[t]{2}{*}{ Correct } & \multirow[t]{2}{*}{ Sens } & \multirow[t]{2}{*}{ Spec } & \multirow[t]{2}{*}{ PPV } & \multirow[t]{2}{*}{ NPV } & \multirow[t]{2}{*}{ LR+ } & \multirow[t]{2}{*}{ LR- } & \multirow[t]{2}{*}{ OR } & \multicolumn{2}{|c|}{ OR 95\% CI } & \multirow{2}{*}{$\begin{array}{l}\text { ROC } \\
\text { AUC }\end{array}$} & \multicolumn{2}{|c|}{ ROC AUC 95\% CI } \\
\hline & & & & & & & & & & & & & & & & Lower & Upper & & Lower & Upper \\
\hline \multirow[t]{5}{*}{ Total } & A & 172 & 8 & 108 & 9 & 47 & 0.320 & 0.674 & 0.145 & 0.923 & 0.295 & 0.830 & 1.891 & 0.926 & 2.04 & 0.74 & 5.62 & 0.674 & 0.587 & 0.760 \\
\hline & B & 172 & 13 & 107 & 10 & 42 & 0.320 & 0.698 & 0.236 & 0.915 & 0.379 & 0.844 & 2.765 & 0.835 & 3.31 & 1.35 & 8.13 & 0.673 & 0.585 & 0.761 \\
\hline & C & 172 & 50 & 104 & 13 & 5 & 0.320 & 0.895 & 0.909 & 0.889 & 0.644 & 0.978 & 8.182 & 0.102 & 80.00 & 27.03 & 236.79 & 0.927 & 0.879 & 0.975 \\
\hline & & 172 & 50 & 103 & 14 & 5 & 0.320 & 0.890 & 0.909 & 0.880 & 0.627 & 0.978 & 7.597 & 0.103 & 73.57 & 25.10 & 215.68 & 0.929 & 0.881 & 0.976 \\
\hline & E & 172 & 50 & 103 & 14 & 5 & 0.320 & 0.890 & 0.909 & 0.880 & 0.627 & 0.978 & 7.597 & 0.103 & 73.57 & 25.10 & 215.68 & 0.903 & 0.855 & 0.952 \\
\hline \multirow[t]{5}{*}{ Female } & A & 56 & 7 & 35 & 4 & 10 & 0.304 & 0.750 & 0.412 & 0.897 & 0.433 & 0.889 & 4.015 & 0.655 & 6.13 & 1.49 & 25.22 & 0.730 & 0.586 & 0.874 \\
\hline & B & 56 & 7 & 35 & 4 & 10 & 0.304 & 0.750 & 0.412 & 0.897 & 0.433 & 0.889 & 4.015 & 0.655 & 6.13 & 1.49 & 25.22 & 0.731 & 0.588 & 0.873 \\
\hline & C & 56 & 14 & 34 & 5 & 3 & 0.304 & 0.857 & 0.824 & 0.872 & 0.550 & 0.963 & 6.424 & 0.202 & 31.73 & 6.66 & 151.14 & 0.920 & 0.843 & 0.997 \\
\hline & D & 56 & 14 & 36 & 3 & 3 & 0.304 & 0.893 & 0.824 & 0.923 & 0.670 & 0.965 & 10.706 & 0.191 & 56.00 & 10.08 & 311.25 & 0.937 & 0.874 & 0.999 \\
\hline & E & 56 & 15 & 33 & 6 & 2 & 0.304 & 0.857 & 0.882 & 0.846 & 0.521 & 0.974 & 5.735 & 0.139 & 41.25 & 7.44 & 228.70 & 0.882 & 0.793 & 0.971 \\
\hline \multirow[t]{5}{*}{ Male } & A & 116 & 7 & 72 & 6 & 31 & 0.328 & 0.681 & 0.184 & 0.923 & 0.362 & 0.827 & 2.395 & 0.884 & 2.71 & 0.84 & 8.72 & 0.668 & 0.564 & 0.772 \\
\hline & B & 116 & 10 & 71 & 7 & 28 & 0.328 & 0.698 & 0.263 & 0.910 & 0.410 & 0.839 & 2.932 & 0.809 & 3.62 & 1.25 & 10.46 & 0.688 & 0.585 & 0.792 \\
\hline & C & 116 & 35 & 70 & 8 & 3 & 0.328 & 0.905 & 0.921 & 0.897 & 0.681 & 0.980 & 8.980 & 0.088 & 102.08 & 25.49 & 408.85 & 0.936 & 0.883 & 0.990 \\
\hline & D & 116 & 35 & 70 & 8 & 3 & 0.328 & 0.905 & 0.921 & 0.897 & 0.681 & 0.980 & 8.980 & 0.088 & 102.08 & 25.49 & 408.85 & 0.936 & 0.882 & 0.990 \\
\hline & E & 116 & 35 & 70 & 8 & 3 & 0.328 & 0.905 & 0.921 & 0.897 & 0.681 & 0.980 & 8.980 & 0.088 & 102.08 & 25.49 & 408.85 & 0.914 & 0.856 & 0.973 \\
\hline \multirow[t]{5}{*}{$<65$ years } & A & 93 & 7 & 58 & 5 & 23 & 0.323 & 0.699 & 0.233 & 0.921 & 0.400 & 0.841 & 2.940 & 0.833 & 3.53 & 1.02 & 12.26 & 0.703 & 0.591 & 0.814 \\
\hline & B & 93 & 11 & 57 & 6 & 19 & 0.323 & 0.731 & 0.367 & 0.905 & 0.466 & 0.863 & 3.850 & 0.700 & 5.50 & 1.79 & 16.89 & 0.721 & 0.604 & 0.838 \\
\hline & C & 93 & 27 & 57 & 6 & 3 & 0.323 & 0.903 & 0.900 & 0.905 & 0.682 & 0.976 & 9.450 & 0.111 & 85.50 & 19.86 & 368.01 & 0.918 & 0.843 & 0.993 \\
\hline & D & 93 & 27 & 57 & 6 & 3 & 0.323 & 0.903 & 0.900 & 0.905 & 0.682 & 0.976 & 9.450 & 0.111 & 85.50 & 19.86 & 368.01 & 0.915 & 0.839 & 0.991 \\
\hline & E & 93 & 27 & 57 & 6 & 3 & 0.323 & 0.903 & 0.900 & 0.905 & 0.682 & 0.976 & 9.450 & 0.111 & 85.50 & 19.86 & 368.01 & 0.929 & 0.868 & 0.990 \\
\hline \multirow[t]{5}{*}{$>65$ years } & A & 79 & 4 & 49 & 5 & 21 & 0.316 & 0.671 & 0.160 & 0.907 & 0.270 & 0.834 & $=1.728$ & 0.926 & 1.87 & 0.46 & 7.65 & 0.701 & 0.579 & 0.823 \\
\hline & B & 79 & 4 & 49 & 5 & 21 & 0.316 & 0.671 & 0.160 & 0.907 & 0.270 & 0.834 & 1.728 & 0.926 & 1.87 & 0.46 & 7.65 & 0.706 & 0.587 & 0.825 \\
\hline & $\mathrm{C}$ & 79 & 20 & 51 & 3 & 5 & 0.316 & 0.899 & 0.800 & 0.944 & 0.755 & 0.957 & 14.400 & 0.212 & 68.00 & 14.84 & 311.50 & 0.957 & 0.912 & 1.001 \\
\hline & D & 79 & 19 & 51 & 3 & 6 & 0.316 & 0.886 & 0.760 & 0.944 & 0.746 & 0.948 & 13.680 & 0.254 & 53.83 & 12.22 & 237.11 & 0.958 & 0.916 & 1.001 \\
\hline & E & 79 & 20 & 51 & 3 & 5 & 0.316 & 0.899 & 0.800 & 0.944 & 0.755 & 0.957 & 14.400 & 0.212 & 68.00 & 14.84 & 311.50 & 0.875 & 0.796 & 0.953 \\
\hline \multirow[t]{5}{*}{ PCI } & A & 123 & 12 & 81 & 7 & 23 & 0.285 & 0.756 & 0.343 & 0.920 & 0.405 & 0.899 & 4.310 & 0.714 & 6.04 & 2.13 & 17.10 & 0.680 & 0.565 & 0.795 \\
\hline & B & 123 & 12 & 81 & 7 & 23 & 0.285 & 0.756 & 0.343 & 0.920 & 0.405 & 0.899 & 4.310 & 0.714 & 6.04 & 2.13 & 17.10 & 0.677 & 0.561 & 0.793 \\
\hline & C & 123 & 30 & 80 & 8 & 5 & 0.285 & 0.894 & 0.857 & 0.909 & 0.599 & 0.976 & 9.429 & 0.157 & 60.00 & 18.19 & 197.92 & 0.909 & 0.839 & 0.980 \\
\hline & D & 123 & 29 & 81 & 7 & 6 & 0.285 & 0.894 & 0.829 & 0.920 & 0.622 & 0.971 & 10.416 & 0.186 & 55.93 & 17.36 & 180.20 & 0.913 & 0.847 & 0.980 \\
\hline & E & 123 & 30 & 79 & 9 & 5 & 0.285 & 0.886 & 0.857 & 0.898 & 0.570 & 0.975 & 8.381 & 0.159 & 52.67 & 16.33 & 169.90 & 0.897 & 0.835 & 0.959 \\
\hline CABG & A & 49 & 7 & 25 & 4 & 13 & 0.408 & 0.653 & 0.350 & 0.862 & 0.547 & 0.736 & 2.538 & 0.754 & 3.37 & 0.83 & 13.64 & 0.711 & 0.560 & 0.862 \\
\hline & B & 49 & 7 & 24 & 5 & 13 & 0.408 & 0.633 & 0.350 & 0.828 & 0.491 & 0.728 & 2.030 & 0.785 & 2.58 & 0.68 & 9.79 & 0.691 & 0.537 & 0.844 \\
\hline & C & 49 & 19 & 27 & 2 & 1 & 0.408 & 0.939 & 0.950 & 0.931 & 0.868 & 0.975 & 13.775 & 0.054 & 256.50 & 21.67 & 3035.99 & 0.991 & 0.973 & 1.008 \\
\hline
\end{tabular}




\begin{tabular}{|c|c|c|c|c|c|c|c|c|c|c|c|c|c|c|c|c|c|c|c|c|}
\hline & & \multirow[t]{2}{*}{$\mathbf{n}$} & \multirow[t]{2}{*}{ TP } & \multirow[t]{2}{*}{ TN } & \multirow{2}{*}{\multicolumn{2}{|c|}{ FP FN }} & \multirow[t]{2}{*}{ a priori } & \multirow[t]{2}{*}{ Correct } & \multirow[t]{2}{*}{ Sens } & \multirow[t]{2}{*}{ Spec } & \multirow[t]{2}{*}{ PPV } & \multirow[t]{2}{*}{ NPV } & \multirow[t]{2}{*}{ LR+ } & \multirow[t]{2}{*}{ LR- } & \multirow[t]{2}{*}{ OR } & \multicolumn{2}{|c|}{ OR 95\% CI } & \multirow{2}{*}{$\begin{array}{l}\text { ROC } \\
\text { AUC }\end{array}$} & \multicolumn{2}{|c|}{ ROC AUC 95\% CI } \\
\hline & & & & & & & & & & & & & & & & Lower & Upper & & Lower & Upper \\
\hline & $\mathrm{D}$ & 49 & 20 & 28 & 1 & 0 & 0.408 & 0.980 & 1.000 & 0.966 & 0.932 & 1.000 & 29.000 & 0.000 & $\mathrm{NaN}$ & $\mathrm{NaN}$ & $\mathrm{NaN}$ & 0.999 & 0.996 & 1.003 \\
\hline & $\mathrm{E}$ & 49 & 20 & 24 & 5 & 0 & 0.408 & 0.898 & 1.000 & 0.828 & 0.734 & 1.000 & 5.800 & 0.000 & $\mathrm{n} / \mathrm{a}$ & $\mathrm{n} / \mathrm{a}$ & $\mathrm{n} / \mathrm{a}$ & 0.905 & 0.816 & 0.995 \\
\hline \multirow[t]{3}{*}{ No MI in history } & A & 136 & 6 & 93 & 3 & 34 & 0.294 & 0.728 & 0.150 & 0.969 & 0.455 & 0.868 & 4.800 & 0.877 & 5.47 & 1.30 & 23.10 & 0.667 & 0.564 & 0.769 \\
\hline & $\mathrm{C}$ & 136 & 35 & 86 & 10 & 5 & 0.294 & 0.890 & 0.875 & 0.896 & 0.593 & 0.976 & 8.400 & 0.140 & 60.20 & 19.19 & 188.83 & 0.925 & 0.868 & 0.981 \\
\hline & $\mathrm{E}$ & 136 & 35 & 85 & 11 & 5 & 0.294 & 0.882 & 0.875 & 0.885 & 0.570 & 0.976 & 7.636 & 0.141 & 54.09 & 17.51 & 167.12 & 0.884 & 0.821 & 0.946 \\
\hline \multirow[t]{3}{*}{ MI in history } & A & 36 & 9 & 17 & 4 & 6 & 0.417 & 0.722 & 0.600 & 0.810 & 0.616 & 0.799 & 3.150 & 0.494 & 6.38 & 1.42 & 28.60 & 0.819 & 0.681 & 0.957 \\
\hline & $\mathrm{C}$ & 36 & 14 & 20 & 1 & 1 & 0.417 & 0.944 & 0.933 & 0.952 & 0.909 & 0.966 & 19.600 & 0.070 & 280.00 & 16.12 & 4863.44 & 0.994 & 0.977 & 1.010 \\
\hline & E & 36 & 15 & 18 & 3 & 0 & 0.417 & 0.917 & 1.000 & 0.857 & 0.781 & 1.000 & 7.000 & 0.000 & $\mathrm{NaN}$ & $\mathrm{NaN}$ & $\mathrm{NaN}$ & 0.957 & 0.898 & 1.016 \\
\hline
\end{tabular}

$\mathrm{n}=$ number of cases; $\mathrm{TP}=$ true positives; $\mathrm{TN}=$ true negatives; $\mathrm{FP}=$ false positives; $\mathrm{FN}=$ false negatives; a priori $=$ a priori probability of stenosis; Correct $=$ fraction of correctly predicted cases; $\mathrm{Sens}=$ sensitivity; $\mathrm{Spec}=$ specificity; $\mathrm{PPV}=$ positive predictive value; $\mathrm{NPV}=$ negative predictive value; $\mathrm{LR}+=$ positive likelihood ratio; $\mathrm{LR}-=$ negative likelihood ratio; $\mathrm{OR}=$ odds ratio; $\mathrm{ROC}$ AUC = receiver operating curve area under the curve (for continuous severity score and probabilities from logistic regression models); $95 \% \mathrm{CI}=95 \%$ confidence interval; Lower $=$ Lower boundary of 95\% CI; Upper = Upper boundary of 95\% CI; NaN = Not a number; $\mathrm{MI}=$ Myocardial infarction; $\mathrm{PCI}=$ percutaneous coronary intervention; $\mathrm{CABG}=$ coronary artery bypass grafting

The severity score ranged from 0 to 11.5 , mean 2.9 $(+/-2.8)$, with $62.8 \%$ of all patients having a severity score of less than 4 . The severity score was significantly higher for patients with relevant coronary stenosis as diagnosed at angiography than for patients without stenosis $(5.6+/-2.1$ vs. $1.7+/-2.2$; $\mathrm{p}<0.01$; Figure 1). For the association between severity score and coronary stenosis, the area under the ROC curve was calculated to be 0.903 [0.855-0.952] (Figure 2). The coordinates of the curve indicated that a cut-off of 4.0 provided the best combination of sensitivity and specificity for the prediction of coronary stenosis from the 3DMP test (as was pre-defined by the manufacturer).

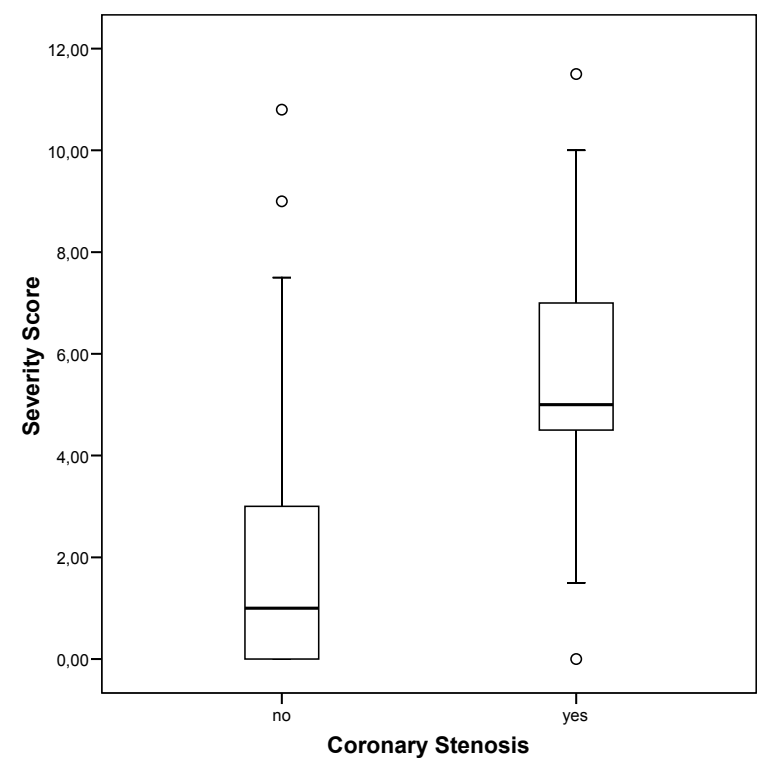

Figure 1. Severity score versus coronary stenosis as diagnosed by angiography. Boxplots of severity score. Circles denote outliers.
Patients without coronary stenosis had a severity score below 4.0 significantly more frequently than those with stenosis $(\mathrm{p}<0.01)$, with $89 \%$ of all cases being correctly classified (OR 73.57 [25.10-215.68]). The results listed in table 4 indicate a sensitivity of $90.9 \%$ and specificity of $88 \%$ for the 3DMP test in the prediction of coronary stenosis (positive predictive value $=0.627$, negative predictive value $=0.978) . \mathrm{A}$ positive likelihood ratio of over 7 and a negative likelihood ratio of 0.1 indicate a good to strong diagnostic value for this test (Table 3).

Sensitivity and specificity did not vary significantly between gender, age groups, or type of revascularization, although sensitivity was especially high in patients after CABG, and specificity in older patients (Table 3). Analysis of ROC also showed that for each subgroup, the best cut-off was 4.0 (Figure 2).

In a logistic regression model, the addition of all risk factors did not significantly improve the classification of coronary stenosis $(89.5 \%$ correct; OR 80.00 [27.03-236.79]). When information about MI history was added to this model again the classification, performance did not change markedly (89\% correct; OR 73.57 [25.10-215.68].

The ROC AUC for a regression model with all risk factors, all risk factors plus information about $\mathrm{MI}$ history, the severity score alone, a regression model with the severity score plus all risk factors, and a regression model with the severity score plus all risk factors and information about MI history were 0.674 [0.587-0.760], 0.673 [0.585-0.761], 0.903 [0.855-0.952], 0,927 [0.879-0.975], and 0.929 [0.881-0.976] respectively (Figure 3). Similar results could be found for each gender and age group (Table 3). 


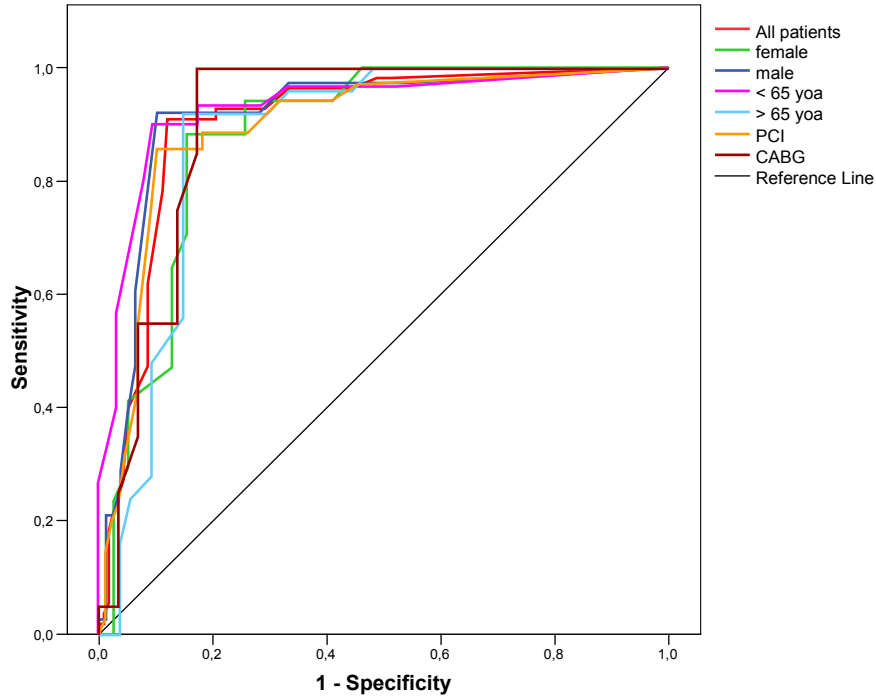

Figure 2. ROC curves for severity score for the detection of coronary stenosis for different gender, age groups, and type of revascularization. yoa $=$ years of age.

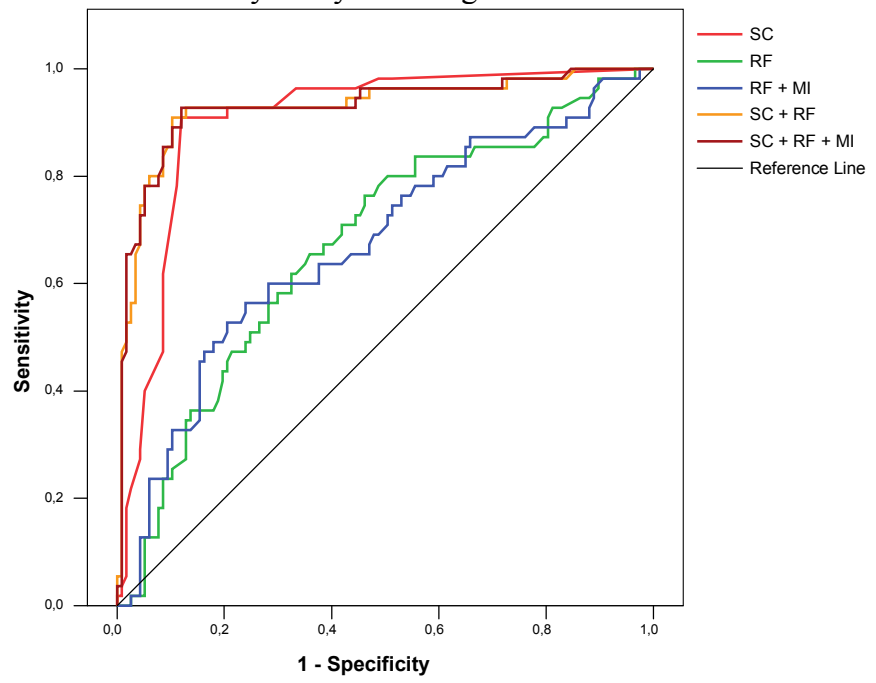

Figure 3. ROC curves of severity score alone ("SC"), risk factors (logistic regression model, "RF"), risk factors and $\mathrm{MI}$ history (logistic regression, "RF + MI"), risk factors plus severity score (logistic regression model, "SC + RF"), and risk factors plus severity score and MI history (logistic regression model, "SC + RF+ MI"), for detecting coronary stenosis.
Table 4: Prediction of coronary stenosis by severity score (cut-off 4.0).

\begin{tabular}{|c|c|c|c|c|}
\hline & & \multicolumn{2}{|c|}{ Prediction Cut-off 4.0 } & Total \\
\hline Coronary Stenosis & no & 103 & 14 & 117 \\
\hline & & $59.9 \%$ & $8.1 \%$ & $68.0 \%$ \\
\hline \multirow{2}{*}{ Total } & yes & 5 & 50 & 55 \\
\hline & & $2.9 \%$ & $29.1 \%$ & $32.0 \%$ \\
\hline & & 108 & 64 & 172 \\
\hline & & $62.8 \%$ & $37.2 \%$ & $100.0 \%$ \\
\hline
\end{tabular}

If patients with history of MI were excluded the diagnostic performance of 3DMP did not change significantly with $88.2 \%$ of these patients correctly classified (details in Table 3).

To further evaluate performance of 3DMP, sensitivity and specificity were assessed at different cut-offs for severity (Table 5). This comparison also showed that a cut-off of 4.0 provided the best compromise of sensitivity and specificity. As the negative predictive value at a cut-off of 4.0 is already high and increases only slightly with lower cut-offs, a value of 4.0 may also be suitable for screening in this patient population.

A second 3DMP test was performed on 38 patients within 4 hours of the first test and before angiography. The test results were identical in 32 patients. In only 1 patient was the difference in severity scores greater than 1 and in only 2 patients would this difference have led to a change in classification (4.0 and 3.0 for the first test, 3.0 and 4.0 for the second test).

Verification after the end of the data acquisition confirmed that locally stored and transmitted ECG data were identical for all recordings.

Table 5: Prediction of coronary stenosis by severity score at different cut-offs for total population $(\mathrm{n}=172$, a priori probability of stenosis $=0.372$ ).

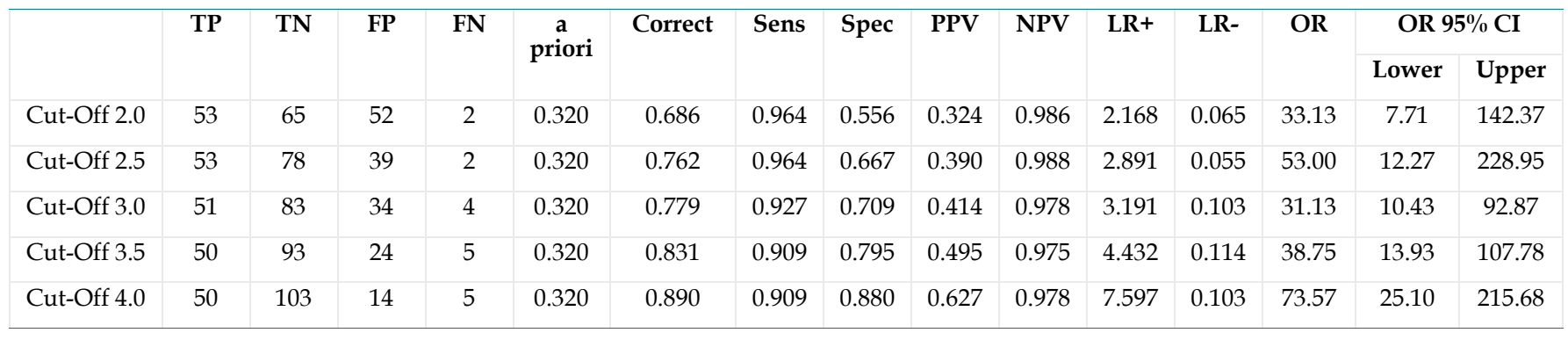




\begin{tabular}{|l|c|c|c|c|c|c|c|c|c|c|c|c|c|c|}
\hline & TP & TN & FP & FN & $\begin{array}{c}\text { a } \\
\text { priori }\end{array}$ & Correct & Sens & Spec & PPV & NPV & LR+ & LR- & OR & OR 95\% CI \\
\hline
\end{tabular}

$\mathrm{TP}=$ true positives; $\mathrm{TN}=$ true negatives; $\mathrm{FP}=$ false positives; $\mathrm{FN}=$ false negatives; correct $=$ fraction of correctly predicted cases; Sens $=$ sensitivity; $\mathrm{Spec}=$ specificity $; \mathrm{PPV}=$ positive predictive value; $\mathrm{NPV}=$ negative predictive value; $\mathrm{OR}=$ odds ratio; $95 \% \mathrm{CI}=95 \%$ confidence interval; Lower $=$ Lower boundary of $95 \%$ CI; Upper $=$ Upper boundary of $95 \%$ CI

\section{Discussion}

The age and gender distributions in the studied patient group match those of patients with symptomatic coronary artery disease reported in the literature [25]. Also the distribution between post-CABG and post-PCI patients corresponds to the official numbers reported for these procedures in most developed countries [1]. The incidence of clinically identified risk factors for CAD among the studied patients was high across the entire study group. The calculated relative risks for symptomatic CAD resulting from the risk factors in the study group is in the range of what is reported in the literature from larger epidemiologic studies [14, 15, 16, 17].

The overall sensitivity of $90.9 \%$ and specificity of $88 \%$ of the 3DMP device are in line with results from a study of 3DMP in patients with CAD but without previous revascularization done at the same center in parallel [22]. Similar performance was also reported from another earlier study, although the results were based on a quantitative assessment of ischemia by the 3DMP system [21]. The quantitative severity score used in the current study was not available at that time.

Resting ECG analysis, including that of the 12-lead ECG, typically has significantly less sensitivity in detecting ischemia. Clinical studies report a wide range of sensitivity from $20 \%$ to $70 \%$ for acute myocardial infarction (AMI) and typically less for hemodynamically significant $\mathrm{CAD}[9,26]$.

Diagnostic yield from the ECG can be improved by exercise testing. Exercise ECG has a reported specificity of over $80 \%$ under ideal conditions. Clinically, however, the sensitivity is typically not better than $50-60 \%$ and shows significant gender bias $[27,28,29,30]$. Performance of exercise ECG testing can further be enhanced by multivariate analysis of ECG and clinical variables. First studies into computerized, multivariate exercise ECG analysis showed good to excellent sensitivity in men and women ( $83 \%$ and $70 \%$, respectively) and specificity $(93 \%, 89 \%)[31,32]$. These results were confirmed by a second group of researchers [33] and are similar to our findings with 3DMP. Other researchers used different statistical approaches and models of multivariate stress ECG analysis with different sets of variables included in the models [34, 35, 36, 37]. While these approaches provided significantly better diagnostic performance than standard exercise ECG testing, it appears that none of these methods has been implemented in broad clinical practice or a commercial product. It should also be noted that none of the above studies included patients with previous coronary revascularization.

In a comprehensive systematic review of 16 prospective studies myocardial perfusion scintigraphy showed better positive and negative likelihood ratios than exercise ECG testing [38]. But wide variation between studies was reported with positive LR ranging from 0.95 to 8.77 and negative LR from 1.12 to 0.09 . Another review of stress scintigraphy studies showed similar results with a diagnostic accuracy of $85 \%$ by wide variation between studies (sensitivity $44 \%-89 \%$, specificity $89 \%-94 \%$, for $2+$ vessel disease) [39]. In one study the combination of stress ECG testing with myocardial scintigraphy using multivariate analysis provided only limited improvement of diagnostic accuracy [40].

Stress echocardiography performed by experienced investigators may provide better sensitivity and specificity than does stress ECG. Numerous studies into exercise echocardiography as a diagnostic tool for CAD have been done. Reported sensitivities range from $31 \%$ to over $90 \%$ and specificities from $46 \%$ to nearly $100 \%$ [41, 42, 43]. With experienced investigators, sensitivities of over $70 \%$ and specificities better than $85 \%$ can be expected.

While the reported diagnostic performance of stress echocardiography, myocardial scintigraphy and stress scintigraphy for the identification of patients with hemodynamically relevant coronary restenosis, graft stenosis or denovo stenosis seems to be similar to that we found for 3DMP, these imaging modalities can provide additional information such as spatial localization that the 3DMP method cannot.

In contrast to the study in patients without previous revascularization from the same center there were no significant differences with respect to 
sensitivity or specificity attributable to gender or age [22]. This may be due to selection effects, or just to the smaller sample size.

The odds ratio for CAD was 2.04 [0.74-5.62] in a logistic regression model using the risk factors identified clinically in this patient group. This is less than in patients without previous revascularization in the same setting investigated with the same methodology [22]. But it is in concordance with large epidemiological studies, although these studies did not specifically investigate patients after coronary revascularization $[14,15,16,17]$. Still, this model could predict coronary stenosis only with a sensitivity of $14.5 \%$ which is markedly less than for the severity score. Adding all risk factors, gender, age, and type of revascularization to the severity score in a logistic regression model improved prediction of CAD only marginally (OR 73.57 [25.10-215.68] vs. OR 80.00 [27.03-236.79]).

The endpoint of this study was the morphological diagnosis of coronary restenosis, de-novo stenosis, or graft stenosis in coronary angiography, whereas the investigated electrophysiologic method (3DMP) assesses functional changes in electrical myocardial function secondary to changes in coronary blood flow. Therefore, even under ideal conditions a 100\% coincidence between angiographic findings and 3DMP results could not be expected. This is probably true for every electrophysiologic diagnostic method.

Resting and stress ECG in CAD patients primarily focuses on ST-segment analysis and the detection of other conduction abnormalities such as arrhythmias. This is not comparable to the 3DMP approach, which calculates a severity score for CAD from a complex mathematical analysis. A comparison between 3DMP, 12-lead resting ECG, and coronary angiography in another study showed a higher sensitivity and specificity for 3DMP than for 12-lead ECG in the detection of coronary stenosis [21].

One limitation of the present study was that the angiography results were not explicitly quantified using a scoring system [44]. Still, the assessment of coronary lesions in the study set forth herein was consistent between two experienced angiographers who independently evaluated the angiograms. Moreover, the relevance of morphological quantification of coronary stenosis in angiograms has been subject to discussion [45]. Because the target criterion was hemodynamic relevant coronary stenosis, subclinical or subcritical lesions may have been classified as non-relevant. This may have artificially reduced the calculated sensitivity and specificity of the 3DMP method. Another limitation of the study may have been patient recruitment. The patient population represented a convenience sample of revascularization patients from a larger group of consecutive patients scheduled for coronary angiography in a single heart center. While this may limit the generalizability of the patient sample used herein, the demographic distribution of this sample matches well with the distributions reported in the literature for patients with CAD as do the incidence and distribution of risk factors. Finally, 3DMP was compared in this study to angiography but not to any other non-invasive diagnostic technology. Therefore, inference about the potential superiority or inferiority of 3DMP in comparison to other ECG-based methods can only be drawn indirectly from other studies.

In conclusion, the mathematical analysis of the ECG by 3DMP appears to provide sensitivity and specificity for the prediction of relevant restenosis, de-novo stenosis, and graft stenosis as diagnosed with coronary angiography in patients after coronary revascularization that is at least as good as that of standard resting or stress ECG test methods reported in other clinical studies. However, this impression needs to be further confirmed in a direct comparison between such methods.

\section{Acknowledgements}

The authors are extremely grateful to Prof. Hans Joachim Trampisch, Department for Medical Informatics, Biometrics and Epidemiology, Ruhr-University Bochum, Germany, for his critical review of statistical methodology and data analysis; to H. Robert Silverstein, MD, FACC, St. Vincent Hospital, Hartford, CT, USA; and to Eric Fedel, Premier Heart, LLC, Port Washington, NY, USA, for their constructive comments and help with the manuscript, and to Joshua W. Klein, Premier Heart, LLC, Port Washington, NY, USA, and George Powell, Tokyo, Japan, for their thorough and thoughtful language editing.

This study was supported partially by institutional funds and partially by an unrestricted research grant from Premier Heart, LLC. Premier Heart, LLC provided the 3DMP equipment for this work free of charge.

\section{Competing Interests}

Dr. Shen is founder and managing member of Premier Heart, LLC. He is also co-inventor of the web-based 3DMP method. The other authors do not have any disclosures to make. 


\section{References}

1. OECD. OECD Health Data 2005: Statistics and Indicators for 30 Countries. Paris: OECD Publishing, 2005.

2. Bengtson JR, Mark DB, Honan MB, Rendall DS, Hinohara T, Stack RS, Hlatky MA, Califf RM, Lee KL, Pryor DB. Detection of restenosis after elective percutaneous transluminal coronary angioplasty using the exercise treadmill test. Am J Cardiol. 1990; $65: 28-34$.

3. Moses JW, Leon MB, Popma JJ, Fitzgerald PJ, Holmes DR, O'Shaughnessy C, Caputo RP, Kereiakes DJ, Williams DO, Teirstein PS, Jaeger JL, Kuntz RE; SIRIUS Investigators. Sirolimus-eluting stents versus standard stents in patients with stenosis in a native coronary artery. N Engl J Med. 2003; 349: 1315-1323.

4. Stone GW, Ellis SG, Cox DA, Hermiller J, O'Shaughnessy C, Mann JT, Turco M, Caputo R, Bergin P, Greenberg J, Popma JJ, Russell ME; TAXUS-IV Investigators. A polymer-based, paclitaxel-eluting stent in patients with coronary artery disease. N Engl J Med. 2004; 350: 221-231.

5. Braunwald E, Antman EM, Beasley JW, et al. ACC/AHA guideline update for the management of patients with unstable angina and non-ST-segment elevation myocardial infarction--2002: summary article: a report of the American College of Cardiology/American Heart Association Task Force on Practice Guidelines (Committee on the Management of Patients With Unstable Angina). Circulation. 2002; 106: 1893-1900.

6. Gibbons RJ, Abrams J, Chatterjee K, et al. ACC/AHA 2002 guideline update for the management of patients with chronic stable angina--summary article: a report of the American College of Cardiology/American Heart Association Task Force on practice guidelines (Committee on the Management of Patients with Chronic Stable Angina). J Am Coll Cardiol. 2003; 41: 159-168.

7. Mason JJ, Owens DK, Harris RA, et al. The role of coronary angiography and coronary revascularization before noncardiac vascular surgery. JAMA. 1995; 273: 1919-1925.

8. Scanlon PJ, Faxon DP, Audet AM, et al. ACC/AHA guidelines for coronary angiography: executive summary and recommendations. A report of the American College of Cardiology/American Heart Association Task Force on Practice Guidelines (Committee on Coronary Angiography) developed in collaboration with the Society for Cardiac Angiography and Interventions. Circulation. 1999; 99: 2345-2357.

9. Ammar KA, Kors JA, Yawn BP, et al. Defining unrecognized myocardial infarction: a call for standardized electrocardiographic diagnostic criteria. Am Heart J. 2004; 148: 277-284.

10. Salerno SM, Alguire PC, Waxman HS. Competency in interpretation of 12-lead electrocardiograms: a summary and appraisal of published evidence. Ann Intern Med. 2003; 138: 751-760.

11. Hecht HS, Shaw RE, Chin HL, Ryan C, Stertzer SH, Myler RK. Silent ischemia after coronary angioplasty: evaluation of restenosis and extent of ischemia in asymptomatic patients by tomographic thallium-201 exercise imaging and comparison with symptomatic patients. J Am Coll Cardiol. 1991; 17: 670-677.

12. Pirelli S, Danzi GB, Alberti A, Massa D, Piccalo G, Faletra F, Picano E, Campolo L, De Vita C. Comparison of usefulness of high-dose dipyridamole echocardiography and exercise electrocardiography for detection of asymptomatic restenosis after coronary angioplasty. Am J Cardiol. 1991; 67: 1335-1338.

13. Schroeder E, Marchandise B, De Coster P, Brichant C, Mitri K, Pieters D, Kremer R. Detection of restenosis after coronary angioplasty for single-vessel disease: how reliable are exercise electrocardiography and scintigraphy in asymptomatic patients? Eur Heart J. 1989; 10: 18-21.

14. Greenland P, Knoll MD, Stamler J, et al. Major risk factors as antecedents of fatal and nonfatal coronary heart disease events. JAMA. 2003; 290: 891-897.

15. Khot UN, Khot MB, Bajzer CT, et al. Prevalence of conventional risk factors in patients with coronary heart disease. JAMA. 2003; 290: 898-904.

16. Wilson PW, D'Agostino RB, Levy D, et al. Prediction of coronary heart disease using risk factor categories. Circulation. 1998; 97: 1837-1847.

17. Yusuf S, Hawken S, Ounpuu S, et al; INTERHEART Study Investigators. Effect of potentially modifiable risk factors associated with myocardial infarction in 52 countries (the INTERHEART study): case-control study. Lancet. 2004; 364: 937-952.

18. Hurst JW. Current status of clinical electrocardiography with suggestions for the improvement of the interpretive process. Am J Cardiol. 2003; 92: 1072-1079.

19. Eskola MJ, Nikus KC, Voipio-Pulkki LM, et al. Comparative accuracy of manual versus computerized electrocardiographic measurement of J-, ST- and T-wave deviations in patients with acute coronary syndrome. Am J Cardiol. 2005; 96: 1584-1588.

20. Feng G. EKG and EEG Multiphase Information Analysis (A collection of unpublished notes, thesis, papers and published articles from mid seventies to the late eighties translated into English from Chinese). First Edition. New York, NY: American Medical Publishers; 1992.

21. Weiss MB, Narasimhadevara SM, Feng GQ, et al. Computer-enhanced frequency-domain and 12-lead electrocardiography accurately detect abnormalities consistent with obstructive and nonobstructive coronary artery disease. Heart Dis. 2002; 4: 2-12.

22. Grube E, Bootsveld A, Yuecel S, et al. Computerized two-lead resting ECG analysis for the detection of coronary artery stenosis. Int J Med Sci. 2007; 7: 249-263.

23. Altman DG, Bland JM. Statistics Notes: Diagnostic tests 2: predictive values. BMJ. 1994; 309: 102.

24. Deeks JJ, Altman DG. Diagnostic tests 4: likelihood ratios. BMJ. 2004; 329: 168-169.

25. Thom T, Haase N, Rosamond $\mathrm{W}$, et al; American Heart Association Statistics Committee and Stroke Statistics Subcommittee. Heart disease and stroke statistics--2006 update: a report from the American Heart Association Statistics Committee and Stroke Statistics Subcommittee. Circulation. 2006; 113: e85-151.

26. Mant J, McManus RJ, Oakes RA, et al. Systematic review and modelling of the investigation of acute and chronic chest pain presenting in primary care. Health Technol Assess. 2004; 8: $1-158$.

27. Anthony D. Diagnosis and screening of coronary artery disease. Prim Care. 2005; 32: 931-946.

28. Cox JL, Teskey RJ, Lalonde LD, Iles SE. Noninvasive testing in women presenting with chest pain: evidence for diagnostic uncertainty. Can J Cardiol. 1995; 11: 885-890.

29. Curzen N, Patel D, Clarke D, et al. Women with chest pain: is exercise testing worthwhile? Heart. 1996; 76: 156-160.

30. Tak T, Gutierrez R. Comparing stress testing methods. Available techniques and their use in CAD evaluation. Postgrad Med. 2004; 115: 61-70.

31. Detry JM, Robert A, Luwaert RJ, et al. Diagnostic value of computerized exercise testing in men without previous myocardial infarction. A multivariate, compartmental and probabilistic approach. Eur Heart J. 1985; 6: 227-238.

32. Robert AR, Melin JA, Detry JM. Logistic discriminant analysis 
improves diagnostic accuracy of exercise testing for coronary artery disease in women. Circulation. 1991; 83: 1202-1209.

33. Deckers JW, Rensing BJ, Tijssen JG, et al. A comparison of methods of analysing exercise tests for diagnosis of coronary artery disease. Br Heart J. 1989; 62: 438-444.

34. Koide Y, Yotsukura M, Yoshino H, Ishikawa K. A new coronary artery disease index of treadmill exercise electrocardiograms based on the step-up diagnostic method. Am J Cardiol. 2001; 87: 142-147.

35. Lehtinen $\mathrm{R}$, Sievänen $\mathrm{H}$, Uusitalo $\mathrm{A}$, et al. Performance characteristics of various exercise ECG classifiers in different clinical populations. J Electrocardiol. 1994; 27: 11-22.

36. Pruvost P, Lablanche JM, Beuscart R, et al. Enhanced efficacy of computerized exercise test by multivariate analysis for the diagnosis of coronary artery disease. A study of 558 men without previous myocardial infarction. Eur Heart J. 1987; 8: 1287-1294.

37. Rodriguez M, Moussa I, Froning J, et al. Improved exercise test accuracy using discriminant function analysis and "recovery ST slope". J Electrocardiol. 1993; 26: 207-218.

38. Mowatt G, Vale L, Brazzelli M, Hernandez R, Murray A, Scott N, Fraser C, McKenzie L, Gemmell H, Hillis G, Metcalfe M. Systematic review of the effectiveness and cost-effectiveness, and economic evaluation, of myocardial perfusion scintigraphy for the diagnosis and management of angina and myocardial infarction. Health Technol Assess. 2004; 8: 1-207.

39. Elhendy A, Bax JJ, Poldermans D. Dobutamine stress myocardial perfusion imaging in coronary artery disease. J Nucl Med. 2002; 43: 1634-1646.

40. Morise AP, Diamond GA, Detrano R, Bobbio M. Incremental value of exercise electrocardiography and thallium-201 testing in men and women for the presence and extent of coronary artery disease. Am Heart J. 1995; 130: 267-276.

41. Geleijnse ML, Krenning BJ, Soliman OI, et al. Dobutamine stress echocardiography for the detection of coronary artery disease in women. Am J Cardiol. 2007; 99: 714-717.

42. Marwick TH, Shaw L, Case C, Vasey C, Thomas JD. Clinical and economic impact of exercise electrocardiography and exercise echocardiography in clinical practice. Eur Heart J. 2003; 24: 1153-1163.

43. Smart SC, Bhatia A, Hellman R, et al. Dobutamine-atropine stress echocardiography and dipyridamole sestamibi scintigraphy for the detection of coronary artery disease: limitations and concordance. J Am Coll Cardiol. 2000; 36: 1265-1273.

44. Alderman E, Stadius M. The angiographic definitions of the bypass angioplasty re-vascularization investigation. Coron Artery Dis. 1992; 3: 1189-1207.

45. Kuntz RE, Baim DS. Defining coronary stenosis: Newer clinical and angiographic paradigms. Circulation. 1993; 88: 1310-1323. 